\title{
Rise of an alternative majority against opinion leaders
}

\author{
K. Tucci, ${ }^{1}$ J. C. González-Avella, ${ }^{2}$ and M. G. Cosenza ${ }^{1}$ \\ ${ }^{1}$ Grupo de Caos y Sistemas Complejos, Centro de Física Fundamental, Universidad de Los Andes, Mérida, Venezuela. \\ ${ }^{2}$ Departamento de Física, Pontificia Universidade Católica do Rio de Janeiro, Caixa Postal 38071, 22452-970 RJ, Brazil.
}

(Dated: November 6, 2018)

\begin{abstract}
We investigate the role of opinion leaders or influentials in the collective behavior of a social system. Opinion leaders are characterized by their unidirectional influence on other agents. We employ a model based on Axelrod's dynamics for cultural interaction among social agents that allows for non-interacting states. We find three collective phases in the space of parameters of the system, given by the fraction of opinion leaders and a quantity representing the number of available states: one ordered phase having the state imposed by the leaders; another nontrivial ordered phase consisting of a majority group in a state orthogonal or alternative to that of the opinion leaders, and a disordered phase, where many small groups coexist. We show that the spontaneous rise of an alternative group in the presence of opinion leaders depends on the existence of a minimum number of long-range connections in the underlying network. This phenomenon challenges the common idea that influentials are fundamental to propagation processes in society, such as the formation of public opinion.
\end{abstract}

PACS numbers: 89.75.Fb, 87.23.Ge, 05.50.+q.

Keywords: Opinion leaders; Social dynamics; Alternative order.

\section{INTRODUCTION}

Propagation processes describe many important activities in societies, such as opinion formation, epidemic propagation, culture dissemination, viral marketing, and innovation diffusion, and their study is of much interest in social, biological and political sciences [1 [5]. A central argument in the research of these processes has been that the most influential agents - a minority of individuals who influence an exceptional number of their peers - are fundamental to the propagation of behaviors in a society [ 6 - 8 ]. These agents are called opinion leaders, influentials, or spreaders $9-12]$; in opinion dynamics models they are also named zealots, inflexibles, or committed agents [13 -16]. Experimental investigation on a social network (Facebook) revealed that influential individuals are actually less susceptible to influence than noninfluential individuals [17]. The activity of opinion leaders has been considered an important resource in the diffusion of information and marketing strategies in society [18. On the other hand, a common idea in social networks has been that the most connected people are responsible for the largest scale of spreading processes [19 21].

Recently, some works have questioned this so-called 'influentials hypothesis' 22]; for example, it has been argued that social contagion is driven by a critical mass of individuals susceptible to influence rather than by influentials [22]; and that there are circumstances under which the most highly connected or the most central people have little effect on a spreading process 23].

Although the notion of opinion leadership seems clear, precisely how and when the influence of opinion leaders over their environment shapes opinions and trends across entire societies remains an open problem. In this paper we present a dynamical agent-based model to investigate the collective behavior of a social system under the in- fluence of opinion leaders. We define opinion leaders as agents that can affect the state of other agents, but their state remains unchanged; i. e., we assume that the interaction leader-agent is unidirectional. This simplifying assumption expresses the basic nature of the interaction with opinion leaders as described in the literature [6 18]. This also corresponds to the notion of cultural status proposed by Axelrod [5]. The unidirectional interaction dynamics of opinion leaders is similar to that of an external field, or mass media, acting on a social system 26, 27]. Then, opinion leaders can be regarded as distributed mediators or originators of mass media messages in a society $[6]$.

As interaction dynamics, we employ Axelrod's rules for the dissemination of culture among social agents [5], a non-equilibrium model that has attracted much attention from physicists 24 32]. In this model, the interaction rule between agents is such that no interaction takes place for some relative values characterizing the states of the agents. This type of interaction is common in social and biological systems where there is often some bound for the occurrence of interaction between agents, such as a similarity condition for the state variable [33 36].

We show that for low values of the fraction of opinion leaders present, the system is driven towards the opinion state of the leaders. However, above some critical value of the fraction of opinion leaders, we find the nontrivial result that a majority group emerges in the system possessing a state non-interacting - or alternative - with that of the leaders, challenging the influentials hypothesis. When the number of available states for the agents is large, the system reaches a disordered state where many small groups coexist. These three collective phases are characterized on the space of parameters of the system, given by the fraction of opinion leaders and a quantity representing the number of available states. 


\section{SOCIAL DYNAMICS IN THE PRESENCE OF OPINION LEADERS}

We consider a system of $N$ agents located at the nodes of a network. The agents are distributed into two populations: a population $\alpha$ representing opinion leaders having a fixed opinion or cultural state, with size $N_{\alpha}$; and a population $\beta$ of agents capable of changing their states, with size $N_{\beta}$, such that $N_{\alpha}+N_{\beta}=N$. The fraction of opinion leaders is $\rho=N_{\alpha} / N$. Both opinion leaders and agents in $\beta$ are randomly assigned to the nodes in the network. The set of neighbors of an agent $i \in \beta$ is denoted by $\nu_{i}$. The state of agent $i \in \beta$ is given by an $F$-component vector $x_{\beta}^{f}(i),(f=1,2, \ldots, F)$, where each component can take any of the $q$ different values $x_{\beta}^{f}(i) \in\{0,1, \ldots, q-1\}$. On the other hand, opinion leaders share the same state, i.e., if $i \in \alpha, x_{\alpha}^{f}(i)=y^{f}$, where each component $y^{f}$ is fixed and remains invariant during the evolution of the system. At any given time, a selected agent in population $\beta$ can interact with any agent in its neighborhood, which can be either another agent in $\beta$ or an opinion leader in population $\alpha$, in each case following the dynamics of Axelrod's model for cultural influence [5]. As initial condition, each state $x_{\beta}^{f}(i)$ is randomly assigned one of the $q^{F}$ possible vector states with a uniform probability. Then, the dynamics of the system is defined by the following iterative algorithm:

1. Select at random an agent $i \in \beta$ and an agent $j \in \nu_{i}$ whose state we generically denote by $x^{f}(j)$.

2. Calculate the overlap between the states of agents $i$ and $j$, defined as

$$
d(i, j)= \begin{cases}\sum_{f=1}^{F} \delta_{x_{\beta}^{f}(i) y^{f}}, & \text { if } j \in \alpha, \\ \sum_{f=1}^{F} \delta_{x_{\beta}^{f}(i) x_{\beta}^{f}(j)}, & \text { if } j \in \beta .\end{cases}
$$

3. If $0<d(i, j)<F$, with probability $d(i, j) / F$ choose $h$ randomly such that $x_{\beta}^{h}(i) \neq x^{h}(j)$ and set $x_{\beta}^{h}(i)=$ $y^{h}$ if $j \in \alpha$, or $x_{\beta}^{h}(i)=x_{\beta}^{h}(j)$ if $j \in \beta$. If $d(i, j)=0$ or $d(i, j)=1$, the state $x_{\beta}(i)$ does not change.

In this model, opinion leaders can affect the states of other agents, but their state remains unchanged. Thus the dynamical changes of the system occur on the population $\beta$. We shall consider small values of $\rho$ to take into account the observation that opinion leaders constitute a minority in a social system [6 8, 17].

When no opinion leaders are present $(\rho=0)$, a system subject to Axelrod's dynamics reaches a stationary configuration in any finite network, where the agents form domains of different sizes. A domain is a set of connected agents that share the same state. A homogeneous phase in a system is characterized by $d(i, j)=F, \forall i, j$. The coexistence of several domains corresponds to an inhomogeneous or disordered phase in a system. It is known that, on several networks, the system reaches a homogeneous phase for values $q<q_{c}$, and a disordered phase for $q>q_{c}$, where $q_{c}$ is a critical point [24, 25].

We consider two order parameters to characterize the collective behavior of the system under the influence of opinion leaders: the normalized average size of the largest domain in population $\beta$, called $S_{\beta}$; and the normalized average size of the largest domain possessing the state of the opinion leaders in population $\beta$, denoted by $S_{\beta}\left(y^{f}\right)$.

First, we study the model in a fully connected network, where every agent in $\beta$ can interact with any other in the system; i. e., $\nu_{i}=\alpha \cup \beta, \forall i$. In this situation, the fraction of opinion leaders $\rho$ also represents the probability for the agent-opinion leader interactions. For a fully connected network with Axelrod's dynamics, in absence of opinion leaders, the critical value $q_{c}$ depends on the system size as $q_{c} \sim N[32$.

Figure (1a) shows the order parameter $S_{\beta}$ as a function of $q / N_{\beta}$ for different values of the fraction $\rho$. When opinion leaders are absent $(\rho=0)$, population $\beta$ reaches an ordered phase for values $q<q_{c}$, characterized by $S_{\beta} \rightarrow 1$, and a disordered phase for $q>q_{c}$, for which $S_{\beta} \rightarrow 0$. As $\rho$ increases, $S_{\beta}$ exhibits a local minimum at a value $q_{*} / N_{\beta}<q_{c} / N_{\beta}$ that depends on $\rho$. Similarly, the value of $q_{c}$ scales as $q_{c} \sim N_{\beta}=N(1-\rho)$, as seen in Fig. (1a). To elucidate the origin of the local minimum, in Fig. (11b) we show the quantity $\sigma \equiv S_{\beta}-S_{\beta}\left(y^{f}\right)$ as a function of $q / N_{\beta}$, for a fixed value of $\rho$. For $q<q_{*}$, the largest domain in $\beta$ acquires the state of the opinion leaders $y^{f}$, and therefore $\sigma=0$. However, for $q_{*}<q<q_{c}$, the largest domain corresponds to another state non-overlapping with $y^{f}$, i.e., $S_{\beta}>S_{\beta}\left(y^{f}\right)$, and therefore $\sigma>0$. For values $q>q_{c}$ disorder appears in the system, and both $S_{\beta} \rightarrow 0$, $S_{\beta}\left(y^{f}\right) \rightarrow 0$, and thus $\sigma=0$.
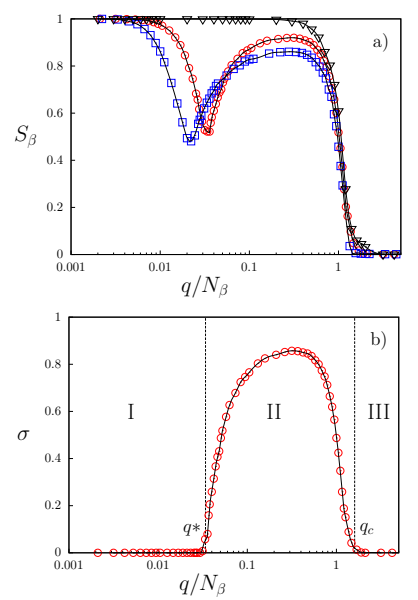

FIG. 1. a) Order parameter $S_{\beta}$ as a function of $q / N_{\beta}$ for parameter values $\rho=0$ (triangles), $\rho=0.05$ (circles), and $\rho=0.1$ (squares). b) Quantity $\sigma=S_{\beta}-S_{\beta}\left(y^{f}\right)$ as a function of $q / N_{\beta}$ for $\rho=0.05$ (circles). Labels I-III refer to the phases shown in the phase diagram of Fig. (3). Fixed parameters are $N=1000, F=5$. Each data point is an average over 50 independent realizations of initial conditions. 
To investigate the role of the opinion leaders on the behavior of the largest domain in population $\beta$, in Fig. (2) we show the dependence of $\sigma$ on the fraction $\rho$, for values of parameters $q_{*}<q<q_{c}$. As $\rho$ increases, a competition ensues between the spontaneous order emerging in population $\beta$ due to the inter-agent interactions and the order being imposed by the opinion leader-agent interaction in $\beta$. For small values of $\rho$, the largest domain in population $\beta$ is driven towards the state of the opinion leaders $y^{f}$, and thus $\sigma=0$. Also as $\rho$ increases, the size of population $\beta$ decreases and, as a consequence, there are fewer agents in $\beta$ whose states share some features with the state $y^{f}$. There is a critical value of the fraction $\rho$ above which the largest domain forming in $\beta$ no longer converges to the state of the opinion leaders $y^{f}$, but reaches a state non-interacting with $y^{f}$, characterized by $\sigma>0$. Thus, above some threshold value of the fraction of opinion leaders, their presence actually promotes the emergence of a majority group in population $\beta$ possessing a state orthogonal to that of the leaders.

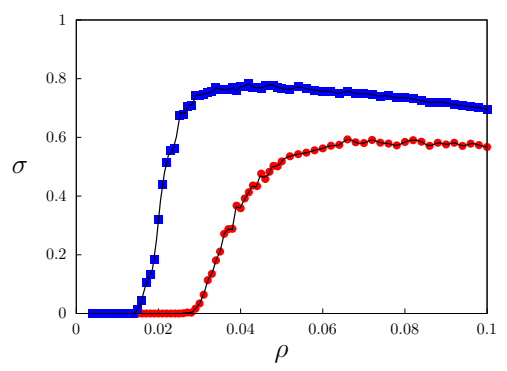

FIG. 2. Quantity $\sigma=S_{\beta}-S_{\beta}\left(y^{f}\right)$ as a function of $\rho$ for parameter values $q=50$ (circles) and $q=100$ (squares). Fixed parameters are $N=1000, F=5$. Each data point is an average over 50 independent realizations of initial conditions.

In Fig. (3) we show the collective behavior of the system subject to the action of opinion leaders on the space of parameters $(q, \rho)$. Three phases can be characterized on this space: (I) an ordered phase imposed by the opinion leaders for $q<q_{*}$, for which $\sigma=0$ and $S_{\beta}=S_{\beta}\left(y^{f}\right) \sim 1$; (II) an ordered phase in a state noninteracting with the state of the opinion leaders (i. e. the overlap between the ordered state and $y^{f}$ is zero) for $q_{*}<q<q_{c}$, for which $\sigma>0$ and $S_{\beta}>S_{\beta}\left(y^{f}\right)$; and (III) a disordered phase for $q>q_{c}$, for which both $S_{\beta} \rightarrow 0$, $S_{\beta}\left(y^{f}\right) \rightarrow 0$, and $\sigma=0$. In phase I, opinion leaders are successful at inducing their cultural state to the largest domain formed in the system. Phase II corresponds to a situation where a group of agents in $\beta$ spontaneously orders in a cultural state non-interacting with that being transmitted by the opinion leaders and emerges as the majority group. In a social context, phase II represents the rise of an alternative group against the imposition of a fixed cultural message or opinion by an influential group, or by a spatially distributed mass media message.

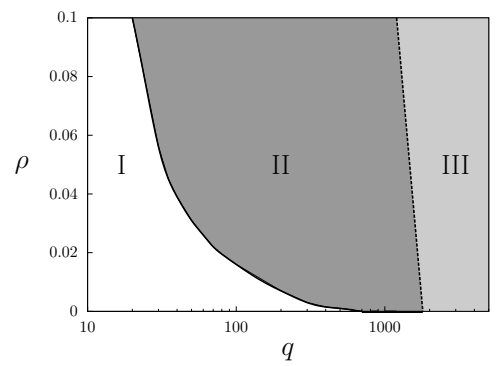

FIG. 3. Phase space on the plane $(q, \rho)$, with fixed parameters $N=1000, F=5$. Regions where phases I-III occur are indicated. The critical boundary between phases II and III follows the relation $q_{c} \sim N(1-\rho)$, which we have verified for different system sizes.

\section{NETWORK OF INTERACTING STATES.}

The collective dynamics of the system associated to each of these phases can be described in terms of the changes in the connectivity of the network of interacting states defined as follows: agents in the system are considered as the nodes of the network, and a link between any two nodes exists if their state variables share at least one component. This network of interacting states is, in general, different from the network of neighbors - a fully connected network in the present case. Let $\beta_{0}$ be the subset of agents in $\beta$ whose state vector initially shares at least one component with the state of the opinion leaders $y^{f}$. This subset has size $N_{\beta_{0}}=N(1-\rho)\left[1-(1-1 / q)^{F}\right]$. Similarly, we denote as $\beta_{0}^{\prime}$ the subset of agents in $\beta$ that initially do not share any component with the state $y^{f}$.

Figure (4) shows the initial (left column) and final (right column) configurations of the interaction network so defined, with a fixed fraction of opinion leaders $\rho$, and for different values of the parameter $q$. Nodes representing opinion leaders in set $\alpha$, as well as agents in subsets $\beta_{0}$ and $\beta_{0}^{\prime}$, are identified by respective colors. In the final configurations, a set of connected agents corresponds to a domain; although, for simplicity, the states associated to different domains are not distinguished by different colors.

Figures (4a) and (4b) correspond to two realizations of phase I. For a small value of $q<q_{*}$ (Fig. (4a)), the size $N_{\beta_{0}}$ is large and both, the probability that the states of agents in $\beta_{0}$ copy additional components of $y^{f}$ and the probability that the states of agents in $\beta_{0}^{\prime}$ acquire components of $y^{f}$ through their interaction with agents in $\beta_{0}$, are high. Eventually, all agents in $\beta$ end up sharing all components of $y^{f}$ and forming one large domain of size $S_{\beta}\left(y^{f}\right)=1$. As $q$ is increased (Fig. (4b)), but still below the value $q_{*}$, those probabilities decrease and not all agents in subset $\beta_{0}^{\prime}$ are able to acquire any component of $y^{f}$. As a consequence, the final interaction network becomes divided into two subgraphs containing agents from $\beta_{0}$ and $\beta_{0}^{\prime}$; the largest subgraph being the largest domain possessing the state $y^{f}$. 


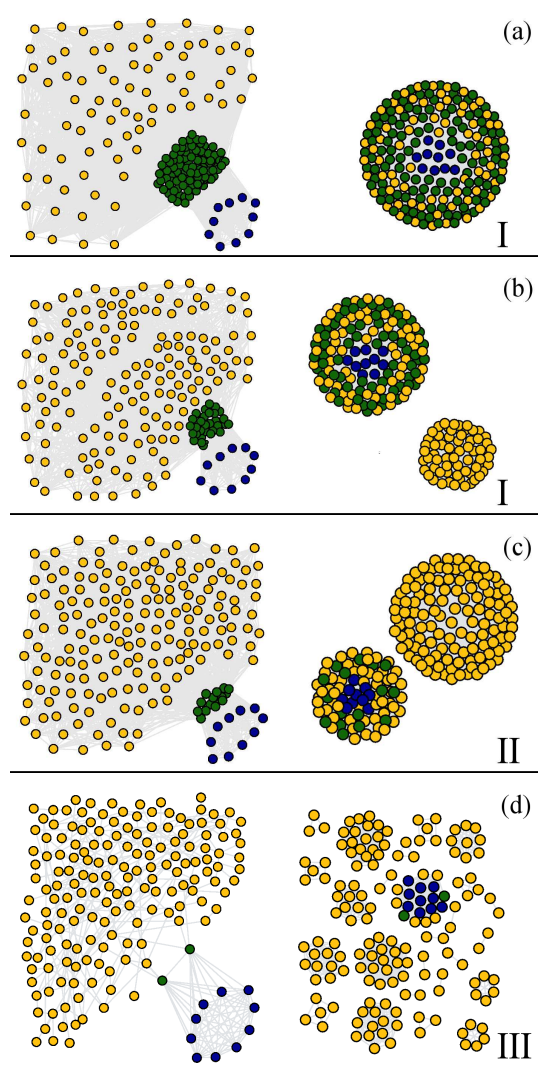

FIG. 4. Initial (left column) and final (right column) configurations of the network of interacting states, for different values of $q$, where a link (gray line) between any two agents exists if their states share at least one component. Opinion leaders (set $\alpha$ ) are depicted in blue color forming a circle; agents that initially share at least one component with the state of the opinion leaders (set $\beta_{0}$ ) are shown in green; and agents that initially do not share any component with the state of opinion leaders (set $\beta_{0}^{\prime}$ ) are drawn in yellow. In the final configurations (right column), a group of connected agents corresponds to a domain. Fixed parameters are $N=200, F=5, \rho=0.05$. (a) $q=20$ (phase I, with $\sigma=0, S_{\beta}\left(y^{f}\right)=1$ ). (b) $q=30$ (phase I, with $\sigma=0, S_{\beta}\left(y^{f}\right)<1$ ). (c) $q=100$ (phase II, $\sigma>0$ ). (d) $q=800$ (phase III, $\sigma=0, S_{\beta} \rightarrow 0$ ).

Figure (4c) shows the configurations of the interaction network for $q_{*}<q<q_{c}$. The final network also consists of two domains, but it is associated to phase II. Now the size of subset $\beta_{0}$ has become too small to contribute efficiently to the transmission of the state of the opinion leaders $y^{f}$ to agents in $\beta_{0}^{\prime}$. Correspondingly, the size of set $\beta_{0}^{\prime}$ is large enough to allow a majority of its agents to form, through their interactions, the largest domain in a state different from $y^{f}$. Finally, Fig. (44) displays the initial and final aspects of the interaction network corresponding to phase III, for $q>q_{c}$. Since $q$ is large, there is a large number of states available to agents in $\beta$ that are non-interacting with the state $y^{f}$. This situation leads to the formation of many small domains and the fragmentation of the network of interacting states.
Thus, Fig. (4) illustrates how the evolution of the system towards its final phase depends on the initial configuration of the interaction network, determined by the size of the subset of agents $\beta_{0}$ whose states initially possess some overlap with the state of the opinion leaders.

\section{LOCAL CONNECTIVITY.}

To investigate the role of the local connectivity of the network on the appearance of phase II, we next consider the dynamics of the system defined on a random network of $N$ nodes having average degree $\bar{k}$. A fully connected network studied above corresponds to the case $\bar{k}=N-1$.

Figure (5) shows the quantity $\sigma$ as a function of $\bar{k}$ for networks of size $N=1000$, for several values of $q$ with other parameters fixed. For values $q>q_{c}$, a disordered state with $\sigma \rightarrow 0$ (both $S_{\beta} \rightarrow 0, S_{\beta}\left(y^{f}\right) \rightarrow 0$ ) is reached for all values of $\bar{k}$ in random networks. However, in connected random networks ( $\bar{k} \geq 8$ for $N=1000)$, for $q_{*}<q<q_{c}$ the largest domain in the network possesses a state orthogonal to $y^{f}$ for which $\sigma>0$. Then, global interactions are not essential for the rise of a majority, alternative group in the presence the opinion leaders; rather this effect depends on the existence of a minimum number of long range connections that is small compared to the size of the network.

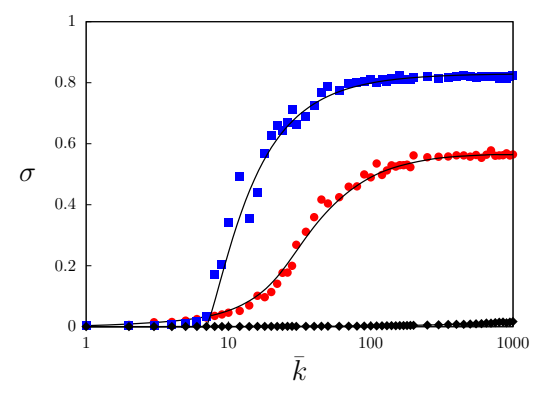

FIG. 5. Quantity $\sigma$ as a function of the average degree $\bar{k}$ of a random network for parameter values: $q=50$ (circles), $q=100$ (squares), $q=2000$ (diamonds). Fixed parameters are $\rho=0.05, N=1000, F=5$. Each data point is an average over 50 independent realizations of initial conditions.

\section{CONCLUSIONS.}

We have investigated the effect of opinion leaders or influentials in the collective behavior of a social system, as well as the role of the network topology in this effect. Our dynamical model is based in Axelrod's rules for cultural interaction among social agents that allow for non-interacting states. Opinion leaders have been characterized by their unidirectional influence on other agents, a behavior similar to that of an external field or mass media acting on a system. In this sense, opinion leaders can be considered as distributed mass media, scattered advertisers, or as a nonuniform field. 
We have found three collective phases in the system depending on parameter values: one ordered phase having the state imposed by the opinion leaders; another nontrivial ordered phase consisting of a large domain on a state orthogonal or alternative to that of the opinion leaders, challenging the influentials hypothesis; and a disordered phase. We have shown that the resulting phase in the system is controlled by the size of the subset of agents whose states initially possess some overlap with the state of the opinion leaders. This explains why a critical mass of agents susceptible to influence, rather than influentials, drives some propagation processes [22].

We have shown that the rise of an alternative, majority group in the presence the opinion leaders depends on the existence of a minimum number of long-range connections in the network. Thus, this phenomenon should be observable in experiments measuring influence, product adoption, or viral marketing in social networks, such as those performed with Facebook users [17].

Our results suggest that the emergence of a selforganized phase with a state different from that of leaders should occur in other non-equilibrium systems possess- ing non-interacting states in their dynamics and enough long-range interactions in their underlying network. This phenomenon could be expected in social and biological systems able to exhibit clustering, aggregation and migration, whose dynamics often possess a threshold condition for interaction 33 36]. Future extensions of this model should include the consideration of diverse interaction rules, the competition of opinion leaders in different or variable states, and the role of complex network topologies.

\section{ACKNOWLEDGMENTS}

This work was supported by project No. C-1906-14-05B from CDCHTA, Universidad de Los Andes, Venezuela. J.C.G-A acknowledges support from CNPq, Brazil, under project No. $150566 / 2015-8$. M. G. C. is grateful to the Senior Associates Program of the Abdus Salam International Center for Theoretical Physics, Trieste, Italy.
[1] E. M. Rogers, Diffusion of Innovations 4th. ed., Free Press, New York, 1995.

[2] M. S. Granovetter, American Journal of Sociology, 83 (1978) 1420,

[3] R. M. Anderson, R. M. May, B. Anderson, Infectious Diseases of Humans: Dynamics and Control (Oxford Science Publications, 1992).

[4] J. Shrager, T. Hogg, B. A. Huberman, Science 236 (1987) 1092.

[5] R. Axelrod, J. Conflict Res. 41 (1997) 203.

[6] E. Katz, and P. F. Lazarsfeld, Personal Influence; the Part Played by People in the Flow of Mass Communications, Glencoe, IL Free Press, 1955.

[7] T. W. Valente, Network Models of the Diffusion of Innovations, Hampton, Cresskill, NJ, 1995.

[8] C. H. Roch, Journal of Politics 67 (2005) 110.

[9] R. K. Merton, in Social Theory and Social Structure, edited by R. K. Merton, Free Press, New York, 1968, p. 441.

[10] G. Weimann, Public Opinion Quarterly 55 (1991) 267.

[11] J. O. Lloyd-Smith, S. J. Schreiber, P. E. Kopp, W. M. Getz, Nature 438 (2005) 355.

[12] J. Borge-Holthoefer, S. Meloni, B. Gonçalves, Y. Moreno, J. Stat. Phys. 151, 383 (2013).

[13] M. Mobilia, A. Petersen, S. Redner, J. Stat. Mech. (2007) P08029.

[14] G. Verma, A. Swami, K. Chan, Physica A 395, (2014) 310.

[15] S. Galam, F. Jacobs, Physica A 381 (2007) 366.

[16] J. Xie, S. Sreenivasan, G. Korniss, W. Zhang, C. Lim, B. K. Szymanski Phys. Rev. E 84, (2011) 011130.

[17] S. Aral, D. Walker, Science 337 (2012) 337.

[18] M. Gladwell, The Tipping Point: How Little Things Can Make a Big Difference, Little, Brown and Company, 2000 .
[19] R. Albert, H. Jeong, A-L. Barabási, Nature 406 (2000) 378.

[20] R. Pastor-Satorras, A. Vespignani, Phys. Rev. Lett. 86 (2001) 3200.

[21] R. Cohen, K. Erez, D. ben-Avraham, S. Havlin, Phys. Rev. Lett. 86 (2001) 3682.

[22] D. J. Watts. P. S. Dodds, Journal of Consumer Research 34(4) (2007) 441.

[23] M. Kitsak, L. K. Gallos, S. Havlin, F. Liljeros, L. Muchnik, H. E. Stanley, H. A. Makse, Nature Phys. 6 (2010) 888.

[24] C. Castellano, M. Marsili, A. Vespignani, Phys. Rev. Lett. 85 (2000) 3536.

[25] K. Klemm, V. M. Eguiluz, R. Toral, M. San Miguel, Phys. Rev. E 67 (2003) 045101(R).

[26] J. C. González-Avella, M. G. Cosenza, K. Tucci, Phys. Rev. E 72 (2005) 065102(R).

[27] J. C. González-Avella, M. G. Cosenza, V. M. Eguíluz, M. San Miguel, New J. Phys. 12 (2010) 013010.

[28] M. N. Kuperman, Phys. Rev. E 73 (2006) 046139.

[29] L. De Sanctis, T. Galla, Phys. Rev. E 79 (2009) 046108.

[30] C. Gracia-Lazaro, L. M. Floria, Y. Moreno, Phys. Rev. E 83 (2011) 056103 .

[31] W. Zhang, C. Lim, S. Sreenivasan, J. Xie, B.K. Szymanski, G. Korniss, Chaos 21 (2011) 025115.

[32] F. Vazquez, S. Redner, Europhys. Lett. 78 (2007) 18002.

[33] G. Deffuant, D. Neau, F. Amblard, G. Weisbuch, Adv. Complex Syst. 3 (2000) 87.

[34] A. S. Mikhailov, V. Calenbuhr, From Cells to Societies: Models of Complex Coherent Action Springer, Berlin, 2002.

[35] G. Weisbuch, G. Deffuant, F. Amblard, J. P. Nadal, Complexity 7 (2002) 55.

[36] R. Hegselmann, K. Krause, J. Artif. Soc. Soc. Simul. 5(3) (2002) 2 . 